\title{
El derecho a la paz en el ámbito educativo: un aporte a la mediación de conflictos
}

\author{
Fecha de recepción : 2019-12-09 • Fecha de aceptación: 2020-04-08 • Fecha de publicación: 2020-09-10
}

Fernando José Castillo'

Universidad Metropolitana del Ecuador (UMET)

fernandocastillo484@hotmail.com

https://orcid.org/0000-0002-0661-448X

Alicia Ramírez de Castillo²

Universidad Metropolitana del Ecuador (UMET)

aliciadecastillo@hotmail.com

https://orcid.org/0000-0002-3615-9166

\section{Resumen}

En todos los sistemas educativos del mundo se plantean estrategias para minimizar los conflictos y la violencia en escuelas y colegios, tratando así de resolver graves confrontaciones entre educandos o estudiantes con docentes, siendo considerada esta una generación violenta. Estas prácticas basadas en valores tienen la finalidad de modelar los caracteres violentos de los alumnos mediante una práctica de mutua convivencia, diálogo y respeto, evitando discrepancias por conflictos raciales, religiosos, políticos, sociales, económicos o culturales. Esta conducta agresiva casi llega a la barbarie ante la incapacidad de convivir en paz, con valores de solidaridad, armonía, cooperación, equidad y no discriminación. Lamentablemente la sociedad se ha acostumbrado a la violencia en el mundo, por lo que hay que realizar cambios obligatorios. Si bien la conducta violenta del alumnado se genera inicialmente en la familia, generando estudiantes problemáticos y desadaptados, hay que realizar las debidas transformaciones en la formación de éstos que permita que se relacionen con la institución educativa mediante la participación e integración en ese ámbito. La esperanza y fe de que se verifiquen esos cambios en las futuras generaciones estudiantiles es deseo que toda la sociedad mundial espera para la consolidación de la paz de la humanidad. 
Palabras clave: derecho a la paz, ámbito educativo, mediación de conflictos.

\section{Abstract}

In all the educational systems of the world strategies are proposed to minimize conflicts and violence in schools and colleges, thus trying to resolve serious confrontations between students and teachers with teachers, considering this a violent generation. These value-based practices are intended to model the violent characters of the students through a practice of mutual coexistence, dialogue and respect, avoiding discrepancies due to racial, religious, political, social, economic or cultural conflicts. This aggressive behavior almost reaches barbarism due to the inability to live together in peace, with values of solidarity, harmony, cooperation, equity and non-discrimination. Unfortunately, society has become accustomed to violence in the world, so mandatory changes have to be made. Although the violent behavior of the students is initially generated in the family, generating problematic and maladjusted students, it is necessary to carry out the necessary transformations in the training of these that allow them to relate to the educational institution through participation and integration in that field. The hope and faith that these changes will take place in future student generations is a desire that the entire world society hopes for the consolidation of the peace of humanity.

Keywords: right to peace, educational field, conflict mediation. 


\section{Introducción}

En las últimas décadas la violencia en el mundo está considerada prácticamente como una curiosa manifestación cultural que, en la actualidad, ya no sorprende a ninguna de las personas integrantes de las sociedades mundiales, puesto que está marcada por hechos de extrema crueldad y saña, de la cual dan cuenta diversas y variadas investigaciones, medios de comunicación impresos o digitales a nivel nacional e internacional con una incomprensible cotidianidad de que esos actos brutales sean responsabilidad del ser humano contra sus semejantes.

Esta situación anómala genera en toda la sociedad una conmoción y una sensación de desesperanza ante la aparente incapacidad de poder convivir en paz como un ideal necesario, por lo que es imperioso realizar los cambios ineludibles dirigidos a la formación de ciudadanos con valores cívicos, como el de autonomía, solidaridad, cooperación y equidad, que asuman el compromiso de participación activa para transformar diversas situaciones conflictivas que puedan presentarse y que tengan como principio fundamental resolver los conflictos a través de mutuos acuerdos y no de manera violenta, además de involucrarse activamente en la construcción de una sociedad basada en elementos democráticos, donde cada persona comparta la responsabilidad y el derecho que se tiene de vivir en un mundo sin odios, discriminaciones, divisiones ni violencia.

Sin embargo, este panorama se agrava cuando a nivel mundial, con sus respectivas excepciones, se observa un sistema educativo con estructuras estereotipadas que parece más bien propiciar la violencia que dar respuestas para disminuir estas conductas, lo que correspondería es formar ciudadanos que dialoguen y trabajen en escenarios enmarcados en una cultura de paz, que piensen y actúen con sensatez, considerando la vida en común y el bienestar colectivo.

Ante tal planteamiento, puede afirmarse que la violencia que se genera en los jóvenes es multicausal e incitada por medios externos pero con fácil acceso a éstos. Es por ello que, desde el interior de los centros educativos, con un buen funcionamiento, una adecuada organización y unas prácticas educativas de comprensión, debidamente aplicadas, se podrán desarrollar tanto en docentes como en los jóvenes las competencias necesarias para aprender a convivir en una cultura enmarcada en la paz, pudiendo ayudar significativamente a controlar este fenómeno.

Bajo este orden orientador en el ámbito educativo, los docentes, el personal directivo, así como padres y representantes, deben adquirir competencias que facilite el aprender y escuchar, que manejen como herramienta la negociación y la mediación de los conflictos y que en el aula de clase se trabaje para enseñar a pensar y actuar teniendo presente los derechos de los estudiantes; puesto que ésta será una solución para tener capacidad de resolver las diferencias que puedan presentarse en el contexto estudiantil.

El desarrollo de programas de formación docente que generen una cultura de paz se hace necesario para abordar de una forma más humanizada las discrepancias y diferencias que existen en una sociedad plural, caracterizada por conflictos raciales, religiosos, políticos, sociales, económicos o culturales, ya que solo así existirán mayores posibilidades de canalizar la agresividad y resolver los antagonismos de forma positiva. Es por ello que se presenta el 
siguiente estudio, con la finalidad de plantear una posible respuesta a la implementación de los medios alternos de solución de conflictos a la problemática de la violencia estudiantil cada vez más acentuada a nivel mundial.

\section{El aprendizaje de valores positivos}

Desde las últimas décadas, de acuerdo con García y Ugarte (1997), aprender un valor significa que la persona es capaz de regular su comportamiento según las normas estipuladas por dicho valor; es modelar la actitud para poder comportarse de una manera determinada ante diferentes objetos, sucesos o personas. Implica verificar que esas virtudes sean bien aprendidas recurriendo a la evaluación como manejo de los conflictos que se presenten mediante la implementación de la cultura de paz en instituciones educativas como manera de determinar en qué medida los valores y las actitudes deseadas están siendo incorporadas a la conducta, su incidencia, el desarrollo y la construcción moral de las personas.

Es por ello que es necesario inculcar en los adolescentes como valor fundamental el respeto por las demás personas, sus orígenes, así como sus ideales, señalamiento que expresa Chitty La Roche (2019): "Respeto... significa valorar a los demás, acatar su autoridad y considerar su dignidad. El respeto se acoge siempre a la verdad; no tolera, bajo ninguna circunstancia, la mentira, y repugna la calumnia y el engaño. El respeto es garantía de transparencia (...).

En este proceso de aprendizaje de valores la educación cumple una función fundamental porque sólo la formación en méritos, cualidades y virtudes de los futuros ciudadanos permitirá la evolución del pensamiento social; aquí el ciclo escolar contribuye a la construcción de nuevas formas de pensar. Según Vázquez, Escámez y García (2012) la educación en valores no es suficiente para que estos cambios se operen en profundidad, la constitución de la cultura de la paz debe venir desde la familia, los medios de comunicación, las empresas, entre otros.

Es necesario formar una conciencia colectiva sobre la necesidad de la paz, eliminando injusticias, buscando una distribución más equitativa de la riqueza y los recursos, asegurando el derecho a la educación en igualdad de condiciones. Enseñar, formar, adiestrar y capacitar son formas de educar para la paz y en valores, porque cuando se enseña e instruye se está transmitiendo consciente e instintivamente una escala de valores, es decir, la educación permite construir actitudes determinadas como la justicia, cooperación, respeto, libertad, solidaridad, autonomía, compromiso, participación y el diálogo. Pero irresponsablemente puede ocurrir que se transmitan antivalores o valores contradictorios a la cultura de paz como la intolerancia, discriminación o violencia.

Cabe destacar que, actualmente, educar para la paz no es inhibir la iniciativa y el interés sino encausar la actividad y el espíritu hacia la consecución de resultados útiles a la sociedad. Representa proporcionar alternativas que favorezcan la comunicación, la convivencia y deseo de participación, fomentando ambientes democráticos en el aula.

\section{En busca de la paz social como futuro deseado}


La gran mayoría de la población que habita en todos los países del orbe, independientemente de los desacuerdos que se puedan presentar entre sus integrantes, se someten a un régimen legal o cuasi legal para dirimir sus diferencias y buscar obtener un beneficio para todas las partes intervinientes en el conflicto, debiéndose a su vez ajustarse a las reglas preestablecidas por los órganos legales correspondientes, aunque es lamentable que no siempre sucede de esta manera, ya que hay personas desadaptadas que no se ajustan a las normativas concebidas, lo que da como consecuencia comportamientos inadecuados que pueden convertirse posteriormente en hechos de violencia, llegando incluso a establecerse como un estado de barbarie.

Es por ello que se debe implementar la mediación como herramienta necesaria para la resolución de conflictos en los centros educativos tomando en cuenta lo señalado por Correa (2016.p.36): "El término mediación se refiere a una puerta de acceso al mejoramiento de las relaciones interpersonales que se encuentran deterioradas por diversas situaciones. Es la vía para atender y mediar aquellas situaciones conflictivas que alteran la buena práctica de los ejercicios sociales". Por lo tanto, llevar a cabo una conciliación o arreglo, ya que negociando acuerdos se puede llegar a una concordia negociada que beneficie a todos y no perjudique a ninguno, es decir, ganar-ganar.

\section{Consecuencias de la violencia en los planteles educativos}

Son muchas las investigaciones que se han referido a esta temática sobre el incremento de la violencia en el ámbito educativo, tales como el bajo rendimiento en la escolaridad por causas psicosociales, lo que genera alumnos problemáticos y desadaptados, además de un clima tenso en el aula, llegando incluso a suscitarse comportamientos antisociales y situaciones estresantes e irregulares.

Ante tales actuaciones, las respuestas habituales de los estudiantes conflictivos en el aula derivan en el aumento de su comportamiento antisocial, tales como acoso sexual, violencia psicológicas mediante amenazas, entre otras faltas o delitos cometidos en el contexto escolar, generando el incremento de los problemas de inseguridad en los centros escolares, como aspectos negativos que obstaculizan el bienestar, la armonía, la estabilidad y la paz en estos entes.

Esta violencia institucional y personal podría parecer una historia impactante y sobrecogedora que ha girado en torno a un sector reducido de la población, de una diversidad de países donde lamentablemente se actúa de manera ilícita en contra de una mayoría que se despliega en la sobrevivencia para acceder a algunos beneficios dentro de su cruda realidad social. Por lo tanto, es imprescindible crear una conciencia ciudadana en esta minoría poblacional, como lo señala Pino (2019.p.1) "la conciencia tiene ramificaciones internas y externas que son dinámicas fruto de las vivencias del sujeto; de allí que es necesario plantear que el sujeto aspira a elevar esa conciencia tanto al mundo interior como al exterior, reconociendo su propia esencia".

Si bien la convivencia humana y su mismo desenvolvimiento vital, por su propia naturaleza, es conflictiva y compleja, independientemente los alumnos siguen interactuando en la comunidad estudiantil en una relación dinámica, aunque vulnerables a lo inesperado del comportamiento constante y permanente de sus compañeros, la mayor de las veces condicionados por el temor, miedo, y hasta el respeto, pero también con la fe y la esperanza de lograr un cambio donde esté 
presente el éxito y no el fracaso; lo que significa una visión positivista de la vida.

\section{La paz como contribución a minimizar conflictos en el ámbito educativo}

El panorama que presenta la percepción de los conflictos como un problema en la educación escolar en los últimos años para lograr cambios conductuales significativos en la población infantil y juvenil, han incidido en los diversos tópicos que han sucedido o acontecen en algunos países, el caso del conflicto en Colombia, de Perú, de América central, de la violencia en Chile y Argentina, de las experiencias policiales en Brasil para combatir la delincuencia, de las pandillas juveniles en Cali, sobre el papel de las fuerzas armadas, el fenómeno de Chiapas, sobre la violencia contra la mujer, sobre los paramilitares y escuadrones de la muerte, las altas tasas de criminalidad en algunas ciudades de América latina, sobre el papel del Estado y una especial atención sobre la literatura, que aborda desde diversas perspectivas la violencia y sus causas (Caballero, 2007, p.305).

Con los cambios conductuales se llevarán a cabo las debidas transformaciones en la formación de la identidad de los niños y jóvenes que tendrá lugar mediante la posición de un rol principal que los alumnos adopten, lo que les permitirá relacionarse con la institución, yendo más allá de sus atributos psicológicos y dependiendo de la aplicación de cinco factores: a) el adecuado escenario familiar; b) los orígenes sociales o entorno familiar del niño y el grupo de edad al cual pertenece; c) la idiosincrasia de la propia institución educativa; d) la presencia de desigualdades económicas; y e) la percepción que el alumno pueda tener de su destino ocupacional en el mundo del trabajo.

Es de fundamental importancia educar apropiadamente para conseguir el perfil requerido, a través de la implementación de diversas estrategias que permitan al docente abordar y manejar adecuadamente los conflictos cuando se presenten contradicciones o inicio de fuertes antagonismos, con el fin de lograr una relación de ganar-ganar, experiencia avalada por Vázquez (2001), quien plantea la necesidad de aprender a canalizar las discrepancias, develando su complejidad y detectando sus causas, lo que, incidiría favorablemente en el ambiente educativo.

Es por ello que tanto docentes, alumnado y personal directivo deben poseer herramientas básicas y utilizar mecanismo apropiados que permitirán conocer el origen de los conflictos en los cuales se pueden ver inmersos, pudiendo enfrentarlos y resolverlos, suprimiendo así una posible escalada y la conformación de una crisis. En este marco, es muy importante que docentes y directivos preparados puedan capacitar a los estudiantes a elegir las maneras de reaccionar ante hostilidades, desarrollando en ellos valores como de solidaridad, habilidades comunicativas basadas en la asertividad, uso de métodos colaborativos como un cambio creativo, para que luego de aprendidas puedan reflexionar acerca de cómo utilizar esas herramientas en la escuela.

Conseguir la meta de este reto no es nada fácil si se parte del hecho de que una buena parte de los adolescentes se desenvuelven en situaciones de conflictos violentos dentro del marco familiar, así como en sus comunidades, viviendo el temor, la ansiedad y la sensación de desesperanza ante un futuro incierto. Por esto, los jóvenes deben ser educados para resolver conflictos de manera asertiva y creativa, formando parte de un proceso de crecimiento personal, potenciando su iniciativa y libertad. Estos cambios deben ser propiciados para las familias en pleno, la comunidad 
e incorporado en el sistema educativo con el cual se estaría comenzando a dar los primeros pasos para el logro de esta transformación.

Con la educación para la paz se refuerza la autoestima para superar el miedo de expresar las propias opiniones y aceptar las ajenas para encontrar soluciones, o simplemente disfrutar de una sencilla conversación entre amigos. Educación para la paz, ha sido siempre un ideal para alcanzar una convivencia adecuada y perenne, pero el significado que se tiene de ella no siempre ha sido el mismo, por lo que se presentan diversas vías quizás contradictorias para encontrarla, debido a que han sido muy diversos los valores que de ella se desprenden.

Desde tiempos inmemoriales se ha presentado la aguda situación de violencia en todos los ámbitos, y se continúa manifestando de diversas maneras: pobreza extrema, formación de pandillas, desempleo, entre otras, lo que genera cada vez más un incremento en la deserción escolar, la urgente necesidad de buscar la conformación de la paz y defender valores como la verdad, justicia y los derechos humanos (Puig, 2008).

Por tal motivo, es importante facilitar las sendas que permitan el manejo constructivo de los desacuerdos que se puedan presentar en las aulas escolares y la manera de planificar diversas actividades para satisfacer las necesidades de los y las jóvenes. Es por ello necesario educar para la paz y el conocimiento para la resolución de los antagonismos que se presentan como un reto para los educadores como principales agentes constructores de la paz, además de la formación para desarrollar habilidades y estrategias que ayuden a sus alumnos a manejar las relaciones interpersonales y enfrentar de manera constructiva posibles conflictos presentados en su vida cotidiana (García y Ugarte, 1997).

De esta manera, la violencia se manifiesta en todos los ámbitos sociales, culturales, económicos y políticos; pero en el aspecto social la violencia se observa como una expresión generalizada que da cuenta de un vacío a ser atendido para poder convivir en paz, siendo los docentes y directivos escolares una pieza principal para introducir los cambios necesarios, por lo tanto, es la escuela como lugar y agente socializador donde se debe tomar conciencia de lo que en realidad aprende el alumno que asiste a las aulas, contra lo que se pretende que se asimile, por lo que se debe asumir la responsabilidad por la cuota de violencia que los jóvenes están manifestando hoy, tanto al interior del centro educativo como en la sociedad en general, y comprometerse en cambiar aquellas estructuras, organizaciones o prácticas educativas, que puedan estimular la violencia en los estudiantes.

En este sentido, la Organización Mundial de la Salud (OMS, 2003) define la violencia como el uso deliberado de la fuerza física o el poder, ya sea en grado de amenaza o efectivo, contra uno mismo, otra persona, un grupo o una comunidad, que cause o tenga muchas probabilidades de causar lesiones, muerte, daños psicológicos, trastornos de muerte, del desarrollo o privaciones. Es por ello que la formación y actualización de conocimientos para la paz de los docentes y personal directivo debe ser permanente y continua, respondiendo así a las necesidades de los sujetos que participan en el proceso educativo; siendo necesario desarrollar competencias no sólo en el conocer, si no en el ser y el convivir, para que puedan aceptar al otro como diferente y portador de iguales deberes y derechos. 
El adecuado adiestramiento de los docentes en el área de educación para la paz, la instrucción en los procesos antiviolencia y la aplicación de métodos de resolución pacífica de conflictos es urgentemente necesaria, ya que al observar, es preocupante como muchos adolescentes de ambos sexos, son seducidos y embaucados en este fenómeno que impera en instituciones de educación media y diversificada, de la cual dan fe, lamentablemente de manera cotidiana, tanto los medios de comunicación escritos, como los audiovisuales.

Por lo tanto, es prioritario cambiar la metodología de la educación escolar en la cual los alumnos puedan expresar sus criterios y hacer saber a docentes y directivos sus inquietudes, por cuanto la mayoría de las actividades se organizan sin la intervención del alumno, donde la práctica educativa es autoritaria, propia de una organización jerárquica y vertical, donde no se promueve la participación y la toma de decisiones consensuada; donde las autoridades escolares poseen la verdad y siempre tienen la razón; donde la posesión de esta verdad es prerrogativa de quienes ostentan el poder; donde se abusa de este dominio conferido institucionalmente, y donde se practica una educación conductista, impidiendo de forma violenta la adquisición y construcción del conocimiento, y la oportunidad de crear y de convivir.

Esta práctica educativa es cultivo para el desarrollo de la violencia escolar, lamentablemente tan común en los países latinoamericanos, por cuanto la imitación de las conductas agresivas que se genera en ellos da como consecuencia el incremento de la brutal espiral. Ya se ha demostrado en diversas investigaciones del área que es tiempo de aceptar que la violencia está en las aulas, en "Comunicación en la Prevención del Conflicto en Instituciones Educativas de Media, Diversificada y Profesional" Arellano (2004) plantea:

En correspondencia con el objetivo de indagar las manifestaciones del conflicto escolar en las instituciones educativas (...) se concluye que existen manifestaciones de violencia escolar como: agresión física a docentes por parte de alumnos, disputas entre alumnos que culminan en enfrentamientos físicos, agresión verbal a docentes, agresión a docentes a través de escritos (grafitis, periódicos, anónimos), hechos de violencia con deterioro de bienes materiales de los docentes, deterioro de la infraestructura y mobiliario; siendo estos hechos de violencia resueltos de manera punitiva a través de levantamiento de actas y suspensiones de clases. Lo cual permite afirmar que en las instituciones objeto de estudio se atacan los conflictos de una manera adversaria (p.203).

Ante la situación descrita es necesario buscar formas de resolver los conflictos, lo cual ya está contemplado en la Constitución de la República del Ecuador (2008) que le otorgó rango constitucional a los Métodos Alternos de Resolución de Conflictos (MARC), cuando en su artículo Art. 97 determina:

Todas las organizaciones podrán desarrollar formas alternativas de mediación y solución de conflictos, en los casos que permita la ley; actuar por delegación de la autoridad competente, con asunción de la debida responsabilidad compartida con esta autoridad; demandar la reparación de daños ocasionados por entes públicos o privados; formular propuestas y reivindicaciones económicas, políticas, ambientales, sociales y culturales; y las demás iniciativas que contribuyan al buen vivir. Se reconoce al voluntariado de acción social 
y desarrollo como una forma de participación social".

Este marco constitucional permite a las instituciones educativas diseñar, implementar y desarrollar proyectos para el manejo de conflictos escolares en la búsqueda de un individuo defensor de la pluralidad de valores y de opciones morales, con un pensamiento abierto plural y democrático, capaz de construir una sociedad donde sea posible vivir en paz, en libertad y en la que el respeto a todos sea la nota dominante.

Actualmente los docentes deben estar conscientes de la realidad que se vive en la escuela, donde se manifiestan, como en el resto de la sociedad, hechos de violencia, haciéndose necesario asumir el reto de enarbolar como proyecto escolar la prevención de los diversos conflictos que se puedan presentar. En función de esto es preciso manejar teóricamente lo relativo a la violencia y las contiendas, además plantear la debida prevención de los antagonismos y rivalidades con lo que se evitaría las hostilidades dentro del ámbito escolar.

Cuando se plantea la temática de la violencia escolar debe enfocarse desde dos perspectivas que están íntimamente relacionadas, la violencia directa o el enfrentamiento que tiene carácter personal, donde se puede utilizar la fuerza física o las palabras como medio para resolver diferencias, mostrándose de diversas maneras: a nivel corporal, psicológico o moral, también se expresa contra objetos y ambiente.

En este sentido, Barbeito y Caireta (2004.p.8) define la violencia como: "la actitud o el comportamiento que constituye una violación o una privación al ser humano de una cosa que le es esencial como persona (integridad física, psíquica o moral, derechos, libertades)". Por otra parte, varios autores coinciden en afirmar que la violencia se da de manera indirecta o estructural y por ende incorporada al sistema económico-social, manifestándose en injusticias sociales, como es el caso de la existencia de una diferencia abismal en la distribución del ingreso, acompañada de marginalidad, analfabetismo, carencia de servicios médicos asistenciales, y dificultad de ascenso socio económico, en altos porcentajes de la población. Lo planteado permite vincular los efectos de la violencia con el hecho de impedir el desarrollo de las potencialidades de los seres humanos. Por su parte, Lederach (2007) señala que:

La violencia (...) es preciso enfocarla como la causa de la diferencia entre lo que las personas podrían ser, pero no son: entre lo actual y lo potencial, en cuanto a lo que se refiere a la realización de una vida mínimamente humana. Por ello tenemos que enfocar la violencia y por consiguiente el tema de la paz en términos de autorrealización, la liberación del individuo de todo lo que le impide gozar de los elementos de la vida... (p.45).

En función de lo expuesto, la violencia no solo puede entenderse como el hecho físico de agresión, sino como todo aquello que impida el desarrollo físico, moral o síquico de una persona hacia su autorrealización, es por esto que la escuela debe cultivar y trabajar en la consolidación de valores, virtudes, conocimientos y hábitos necesarios para la preparación de un ciudadano autónomo, entendido este como el ser capaz de tomar libremente decisiones, capaz de compartir, solidarizarse, colaborar comprometerse y organizarse sin dejarse someter por coacciones, en la búsqueda de una sociedad donde todos podamos vivir en paz. 
Por otro lado, los conflictos escolares genéricamente se refieren a cualquier desacuerdo que se manifiesta en cualquier momento o situación; para su expresión se requiere de dos partes relacionadas, ya sea individuos o grupos divididos por causa de intereses u objetivos percibidos como discordantes.

El conflicto así estimado se concibe como una situación donde se manifiesta una divergencia de necesidades, intereses, propósitos u objetivos contrapuestos o que al menos son percibidos así por las partes involucradas, conllevando esto a que sus pretensiones, deseos e intereses, no puedan lograrse simultáneamente, generándose manifestaciones con diversos grados de intensidad, llegando incluso hasta la violencia. Ante estas manifestaciones distintas lo relevante en todo caso es que las acciones para lograr los objetivos derivadas de los intereses divergentes sean canalizadas a través del uso de métodos alternos que permitan el logro de acuerdos aceptables y positivos para las partes.

Es importante recalcar la imposibilidad para eliminar los conflictos con cualquier iniciativa de naturaleza educativa, dado que forman parte de todo proceso donde interaccione el ser humano. En tal sentido, docentes y directivos pueden ayudar a los jóvenes que aprendan a elegir entre las diferentes maneras de reaccionar ante los debates; desarrollando en ellos habilidades de resolución de problemas, permitiéndoles así considerar los antagonismos no como una crisis sino como una ocasión de cambio creativo. Ante todo, pueden aprender a utilizar esas habilidades que forman parte de su cotidianidad en la escuela.

Ante tal exposición, se puede reflexionar acerca de cómo esta aproximación a la resolución de rivalidades podría aplicarse a los hechos que se generan a nivel de la sociedad y que los jóvenes, cómo constructores del futuro deben afrontar. Hacer estos cambios y aceptar el reto no es tarea fácil, si se parte del hecho de la existencia de muchos adolescentes educados en situaciones de conflicto violento, real o probable, en el marco de sus familias y comunidades, han vivido la ansiedad, temor o sensación de desesperanza ante el futuro y sentimiento de impotencia, ante unas fuerzas más allá de su control; para estos adolescentes aprender a resolver conflictos, de manera asertiva y creativa, puede formar parte de un proceso de crecimiento personal, aunado a un sentimiento de capacidad y seguridad.

En este orden orientador, se observa que el cambio para los docentes y personal directivo tampoco es tarea fácil, enmarcados y formados profesionalmente dentro de un conductismo que castra la iniciativa, creatividad y libertad. Pero es tiempo de trasformaciones y la escuela, con todas las trasformaciones que se están generando en su sistema educativo se están dando los pasos necesarios para su transición definitiva.

Lo expuesto permite afirmar que la educación como factor constructor de una sociedad más justa, menos violenta, más participativa, con mejores posibilidades de crecimiento, debe dirigir el proceso en y para la prevención de los conflictos, lo cual supone expresar las bases del poder tanto propio como ajeno, aprender a entender que, si bien se tienen derechos, también se tienen deberes y que el otro también es sujeto de derechos.

En virtud de lo precedentemente expuesto, este planteamiento, estimula a formar ciudadanos que 
hacen de la convivencia un principio para su vida en sociedad. En este sentido, la prevención a nivel educativo significa poner en marcha un proceso donde se crean las bases para enfrentar cualquier divergencia en el momento en que ésta se produzca, mejorando las relaciones entre los sujetos actuantes del proceso educativo, partiendo de la necesidad de relacionarse con detenimiento, tanto docentes, directivos y alumnos, dándole significado a las experiencias personales, estando conscientes de las limitaciones y potencialidades de cada grupo.

En consecuencia y atendiendo a una actitud positiva, resolver constructivamente los conflictos generados en las instituciones educativas es un reto el cual debe ser asumido por toda la sociedad, pero que afecta en forma directa al personal docente y directivo, los cuales deben poseer las habilidades y los recursos necesarios para afrontar las diversas situaciones conflictivas generadas en estos espacios educativos, en función de lograr resultados positivos en el manejo de los conflictos debiéndose tomar en cuenta los aspectos que puedan estar afectando la armonía y estabilidad del alumnado en la institución, facilitando los medios que permitan canalizarlos a través de la fuerza motivadora, la comunicación abierta, el diálogo, la cooperación, promoviendo de manera constante el aprendizaje de los valores y la cultura de la paz para una mejor convivencia.

\section{Metodología}

Es de gran importancia para todo investigador describir específicamente el tipo de investigación que desarrolla, con la finalidad de definir su contexto o naturaleza, motivar e involucrar al lector en ese mundo investigativo enmarcado en una problemática, que se perfila en la búsqueda de soluciones.

La metodología utilizada en el presente escrito corresponde a la dogmática jurídica, de tipo documental, donde se analizaron diversas normativas jurídicas, artículos de revistas científicas y textos alegóricos, tanto en físicos como digitales, con la finalidad de considerar la realidad planteada, lo que permite profundizar en las características particulares de un determinado hecho, describirlo y precisarlo con el fin de dar respuestas a los objetivos planteados.

En este estudio no se presentan resultados, debido a que sólo se emplean en una investigación de campo, por cuanto ese proceso es de forma inmediata, por tener contacto personal y directo con todas las actividades que se realizan, permitiendo de esta manera el conocimiento de la problemática descrita, La investigación de campo se presenta mediante la manipulación de una variable externa no comprobada, en condiciones rigurosamente controladas, con el fin de describir de qué modo o por qué causas se produce una situación o acontecimiento particular. Como ya se indicó, por el tipo de metodología aplicada, sólo se presentan apreciaciones conclusivas.

\section{Conclusiones}

Es un hecho que la violencia está radicada en las escuelas y colegios lo que se manifiesta en dos vertientes: una estructural que se presenta en la exclusión de la población de niños y adolescentes dentro de un proceso educativo que debería brindar una opción de calidad de vida; y una violencia directa que se manifiesta en la destrucción de las instalaciones educativas, de los bienes 
personales de los docentes, alumnos y de la comunidad, además de las confrontaciones entre los educandos y entre docentes y alumnos. Otra material, donde se puede afirmar que la función del docente está en modelar el carácter social del estudiantado para formarlo como ciudadanos que practiquen los valores de convivencia y que ejerzan su autonomía, incidiendo esto en evitar la generación de una violencia directa presente en las instituciones educativas y en su contexto.

Existe la urgente necesidad de transformar la actuación de docentes y alumnos en la búsqueda de un sistema escolar donde se aprenda desde la interrelación a resolver las discrepancias a través del dialogo, donde el respeto sea parte de la cotidianidad, que se tenga como meta aprender a convivir juntos, por lo que se necesita una escuela que eduque para la paz, que no forme alumnos sumisos ni violentos, sino que su función sea la de integrar individuos a la comunidad, arraigados a los principios democráticos y comprometidos como fundamento de vida en una cultura de paz.

Se puede concluir que los docentes deben desistir de la escuela tradicional y comprometerse con los necesarios cambios, prescindir de ejercer el poder por el poder y transponerlo hacia sus alumnos en la búsqueda de una autodisciplina que les permita desarrollar la autonomía, para iniciar una formación en métodos de resolución de conflicto, que les permita escuchar y no solo oír. Debe también facilitarse desde el aula los medios y los recursos que permitan prevenir el conflicto.

Este análisis permite el suficiente soporte para aseverar que es prioritario el control en las escuelas que están marcadas por la violencia, para poder formar al futuro hombre como ser reflexivo que esté en capacidad de definir las causas de los problemas y proponga caminos de solución a través del consenso y de una búsqueda intencional, metódica y autónoma. 


\section{Referencias}

Arellano, N. (2004). Comunicación en la prevención del conflicto en instituciones educativas de media, diversificada y profesional. Cabimas, estado Zulia. Venezuela.

Asamblea Nacional Constituyente. (2008). Constitución de la República del Ecuador.

Barbeito, C. y Caireta, M. (2004). Cuadernos de Educación para La Paz. Escola de Cultura de Pau (Universidad Autónoma de Barcelona). https://escolapau.uab.cat/img/programas/educacion/publicacion010e.pdf (diciembre 2019)

Caballero, F. (2007). Representación de la violencia en América Latina. Revista de la Facultad de Ciencias Jurídicas y Políticas de la Universidad de Carabobo №3. Valencia. Venezuela.

Correa Requena, J. A. (2016). El rol de la mediación en la solución de los conflictos escolares en los establecimientos educativos de nivel medio en el cantón Huaquillas de la Provincia de El Oro, durante los períodos lectivos 2011-2013 (Master's thesis, Universidad Andina Simón Bolívar, Sede Ecuador).

Chitty La Roche, N. (2019) Del irrespeto y otras humillaciones más. Diario El Nacional, Venezuela. Página de Opinión del 12 de abril de 2019.

García, H., \& Ugarte, D. (2002). Resolviendo Conflictos en la Escuela: manual para maestros. Ministerio de Educación.

Lederach, J. (2007) La violencia escolar y la prevención del conflicto. Orbis: Revista de Ciencias Humanas.

Organización Mundial de la Salud (OMS, 2003). Informe mundial sobre la violencia y la salud. Ginebra.

Pino, J. (2019) Conciencia, convivencia y mundo de la vida. Diario El Nacional, Venezuela. Página de Opinión Pensar Educativo del 03 de enero de 2019. Disponible en:

http//www.el-nacional.com/autores//pensareducativo

Puig, S. M., \& Mir, S. (1994). El derecho penal en el Estado social y democrático de derecho. Editorial Ariel.

Vázquez, F. (2001). La memoria como acción social: relaciones, significados e imaginario. Paidós.

Vázquez, V., Escámez, J., \& García, R. (2012). Educación para el cuidado. Hacia una nueva pedagogía. Revista Complutense de Educación, 26(1), 205-215. 
Copyright (c) 2020 Fernando José Castillo y Alicia Josefina Ramírez de Castillo

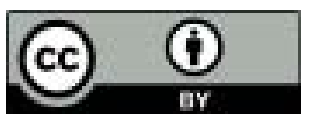

Este texto está protegido bajo una licencia internacional Creative Commons 4.0.

Usted es libre para Compartir-copiar y redistribuir el material en cualquier medio o formato - y Adaptar el documento - remezclar, transformar y crear a partir del material-para cualquier propósito, incluso para fines comerciales, siempre que cumpla las condiciones de Atribución. Usted debe dar crédito a la obra original de manera adecuada, proporcionar un enlace a la licencia, e indicar si se han realizado cambios. Puede hacerlo en cualquier forma razonable, pero no de forma tal que sugiera que tiene el apoyo del licenciante o lo recibe por el uso que hace de la obra.

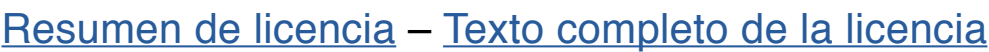

